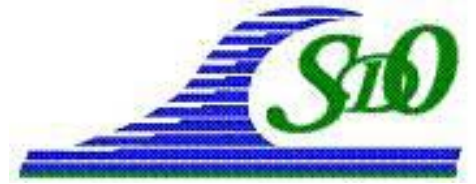

Xìmes Journées Nationales Génie Côtier - Génie Civil

Les Sables d'Olonne, 22-25 juin 2010

DOI:10.5150/jngcgc.2010.032-G @ Editions Paralia CFL

disponible en ligne - http://www.paralia.fr - available online

\title{
Impacts des tempêtes sur la morphologie d'un littoral microtidal : le site du Lido de Sète à Marseillan, Golfe du Lion
}

\section{Mathieu GERVAIS ${ }^{1}$, Yann BALOUIN ${ }^{1}$, Rémi BELON ${ }^{1}, \operatorname{Raphaël~CERTAIN~}^{2}$, Nicolas ROBIN ${ }^{2}$, Serge BERNE ${ }^{2}$}

1. BRGM, Service Géologique du Languedoc-Roussillon, 1039 rue de Pinville, 34000 Montpellier, France.

m.gervais@brgm.fr

2. EA 4218 IMAGES, Université de Perpignan, 52 av Paul Alduy, 66860 Perpignan Cedex, France.

\section{Résumé :}

L'action des tempêtes marines entraîne souvent des réponses morphologiques rapides et des impacts importants sur les littoraux sableux : recul du trait de côte, submersion et érosion de la plage, franchissements et destruction des systèmes dunaires et parfois de l'ensemble du cordon littoral, mais provoque également une importante dynamique des barres sédimentaires d'avant-côte. Dans le cadre du projet européen MICORE (Morphological Impacts and COastal Risks induced by Extreme storm events), des campagnes de mesures sont menées depuis 2008 sur le Lido de Sète à Marseillan, afin de caractériser la dynamique des morphologies sableuses lors des événements énergétiques. L'objectif principal de ces campagnes est d'étudier la variabilité des réponses morphologiques en fonction des différents facteurs de forçages hydrodynamiques (vagues, marée, surcote), atmosphériques (vent, dépression atmosphérique, précipitation) et sédimentaires (morphologie préexistante) lors des tempêtes.

Deux secteurs de plage à morphologies différentes ont été suivis de manière intensive par des techniques conventionnelles de topo-bathymétrie et d'hydrodynamique afin de caractériser leurs réponses respectives aux tempêtes marines. Le secteur sud est caractérisé par la présence de deux barres d'avant-côte quasi-rectilignes et le secteur nord est caractérisé par des morphologies sous-marines fortement tridimensionnelles (deux barres festonnées). Plusieurs tempêtes majeures se sont produites lors de ce suivi (celles du 26 décembre 2008 et du 21 octobre 2009 sont les plus importantes). Elles présentaient des caractéristiques très différentes en terme de hauteurs et périodes de la houle, mais également en terme de niveaux de surcote.

$\mathrm{Au}$ niveau de la plage émergée, la réponse morphologique des deux secteurs est globalement identique et se traduit par des apports massifs de sable vers le haut de plage suite aux évènements les plus extrêmes. Pour la plage immergée, si le schéma classique de recul des barres pendant les tempêtes est observé sur les deux secteurs, la réponse 
tridimensionnelle est très différente : au sud, la barre interne reste rectiligne quels que soient les forçages; au nord, le système alterne entre des situations rectilignes, en croissants, ou en festons rompus, avec des transitions très rapides.

Les évolutions observées sur les deux zones semblent montrer que la cinétique des barres est principalement fonction du niveau d'agitation (Hs atteinte lors de l'évènement de houle). Toutefois, la morphologie préexistante joue un rôle prédominant dans la transition entre les différents types de barres dans la zone $\mathrm{N}$.

Les levés, réalisés après chaque coup de mer significatif $(\mathrm{Hs}>1,5 \mathrm{~m})$ au cours du premier hiver, permettent de caractériser des seuils morphogènes et mettent en évidence l'importance des processus inter-tempêtes. En effet, le rôle des évènements de houle modérée se révèle fondamental dans le retour aux conditions "d'équilibre".

\section{Mots-clés :}

Tempêtes - Morphodynamique - Barres d'avant-côte - Paramètres de houle - Surcote Erosion - Résilience de la plage - Dépôt

\section{Introduction}

Les épisodes météo-marins extrêmes induisent des modifications soudaines et radicales $\mathrm{du}$ cordon sableux littoral. Ils sont souvent associés à de l'érosion du disponible sédimentaire, des reculs du trait de côte, des brèches dans le système dunaire, ou mêmes des percées totales des lidos, de façon parfois irréversible. Les tempêtes provoquent aussi des déplacements importants vers le large des barres sableuses d'avant-côte.

Dans le Golfe du Lion, où des phénomènes érosifs continus s'associent à une forte anthropisation du littoral, ces phénomènes peuvent entraîner des impacts importants sur le milieu et sur les infrastructures ainsi que sur les activités humaines. Dans le contexte du projet européen MICORE (Morphological Impacts and COastal Risks induced by Extreme storm events), des campagnes de mesures morphodynamiques et hydrodynamiques sont menées depuis 2008 sur le Lido de Sète à Marseillan, afin de caractériser la dynamique naturelle des entités morphologiques lors des tempêtes marines ainsi que les impacts induits sur le système littoral.

L'objectif de cet article est de caractériser les réponses morphologiques associées aux évènements très énergétiques (tempêtes « $T$ ») mais aussi celles associées aux épisodes de plus faible agitation («EPFA»), afin d'améliorer la compréhension des facteurs (hydrodynamiques, météorologiques, morphologiques) qui prédéterminent les évolutions lors des coups de mer de différentes ampleurs.

\section{Site d'étude et méthodologie}

Le site d'étude est le lido de Sète à Marseillan, localisé en Languedoc-Roussillon, et séparant l'étang de Thau de la mer Méditerranée. Le marnage est microtidal $(0,19 \mathrm{~m}$ en moyenne à $0,31 \mathrm{~m}$ lors des $\mathrm{VE})$, et le régime de vagues relativement calme $(80 \% \mathrm{des}$ hauteurs significatives Hs $<1 \mathrm{~m}$, bouée DRE de Sète). Deux vents locaux dominent : la 


\section{XI $I^{\text {èmes }}$ Journées Nationales Génie Côtier - Génie Civil}

Les Sables d'Olonne, 22-25 juin 2010

tramontane, prédominante, de direction $\mathrm{O}$ à NNO, et le marin généralement de $\mathrm{S}$ à ESE, Ce vent marin génère au large les épisodes de forte houle, qui sont principalement de direction ESE, mais peuvent également provenir du Sud. A Sète, les houles de tempête annuelles ont une hauteur significative de 4,63 m au large (GLOASGUEN, 1998). L'élévation locale du niveau de la mer lors de ces événements peut atteindre $1 \mathrm{~m}$ sur la plage (0,98 m enregistré le 17/12/1997 dans le port de Sète).
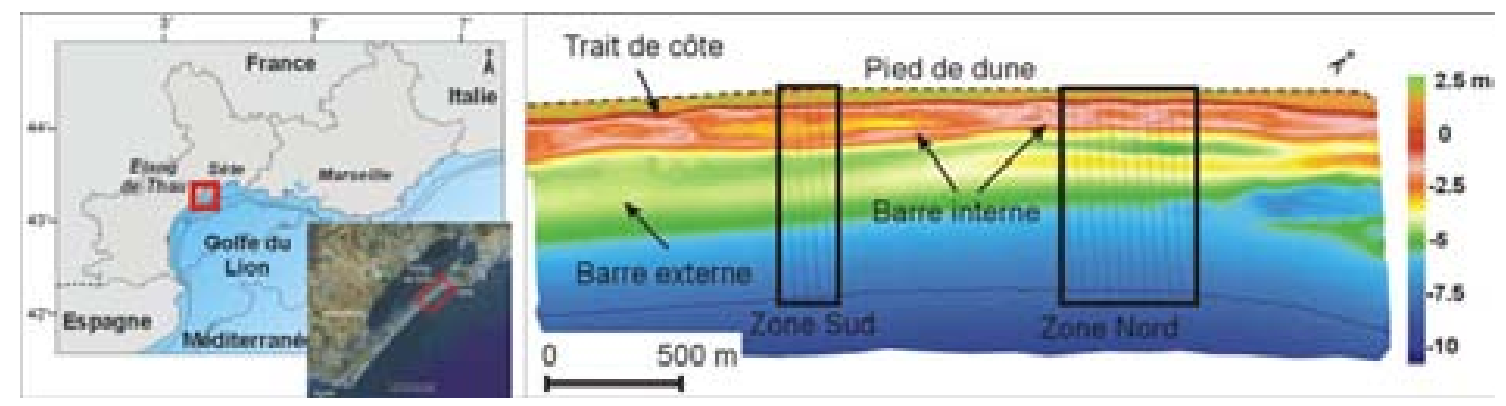

Figure 1. Localisation de la zone d'étude (à gauche) et bathymétrie du 18/11/2008 (à droite) illustrant les zones de suivi haute fréquence Nord et Sud et les morphologies d'avant-côte dans la moitié NE du Lido.

La morphologie de ce littoral est caractérisée par une plage étroite et basse et des morphologies de barres d'avant-côte interne et externe qui sont pseudo-rectilignes à rythmiques (CERTAIN, 2002). Les travaux d'aménagement en cours sur le lido (recul stratégique de la route) ont sensiblement modifié la morphologie du secteur NE avant notre étude, par la reconstruction d'un cordon dunaire étroit, et le rechargement de la plage émergée qui a permis de réalimenter un système d'avant-côte décrit comme fortement déficitaire (CERTAIN, 2002).

Les études menées sur ce secteur (BARUSSEAU \& SAINT-GUILY, 1981; AKOUANGO, 1997 ; CERTAIN, 2002 ; FERRER et al., 2006) ont mis en évidence des évolutions rapides et une dynamique des barres d'avant-côte très importante lors des tempêtes. Celles-ci oscilleraient autour d'une position «d'équilibre» au rythme des épisodes énergétiques (modalité d'Oscillation autour d'une Position d'Equilibre, OPE, AKOUANGO, 1997 ; CERTAIN \& BARUSSEAU, 2005). Dans un contexte temporel plus large, les tempêtes décennales, ou les hivers particulièrement riches en tempêtes, sont responsables d'un soudain recul de la barre externe qui conduit à sa disparition en quelques années (modèle Net Offshore Migration, NOM, observé par CERTAIN, 2002). Le levé réalisé en novembre 2008 a permis de caractériser l'état initial du site avant la première période hivernale. La morphologie était alors caractérisée par (figure 1) :

- Plage émergée : haut de plage à pente très faible (terrasse), berme avec encoche/falaise d'érosion due à la tempête précédent l'étude, croissants de plage formés sur la zone de jet de rive en bas de plage. Sinuosités du trait de côte reflétant les motifs festonnés de la barre interne ; 


\section{Thème 2 - Dynamique sédimentaire et transports des particules}

- Barre interne : barre proche et motifs festonnés $(\lambda=350 \mathrm{~m})$ à l'extrême sud ; barre éloignée, profonde et quasi-linéaire $(\lambda=1450 \mathrm{~m})$ pour la moitié sud restante; barre proche, peu profonde, et festons très marqués $(\lambda=270 \mathrm{~m})$, pour la moitié nord ;

- Barre externe : barre peu marquée, éloignée, profonde, et linéaire pour l'ensemble de la moitié sud; barre plus marquée et plus proche pour toute la partie nord, avec une morphologie festonnée de grande ampleur $(\lambda=1300 \mathrm{~m})$.

Afin de mieux appréhender le rôle de la morphologie sur les évolutions lors des tempêtes, deux secteurs contrastés (parmi ceux décrits juste avant) ont été suivis à haute fréquence sur cette partie NE du lido (figure 1): la zone Sud, présentant 2 barres linéaires profondes (la barre externe très plate semblant en forte dégénérescence), et la zone Nord, où les systèmes de barres sont bien développés, avec des morphologies en croissant bien formées sur la barre interne. De cette manière, la zone sud servira de zone témoin où les barres sont homogènes longitudinalement et où seront quantifiables les processus de mobilisation sédimentaire transverses à la plage, tandis que la zone nord, de part son caractère fortement tridimensionnel, permettra d'apprécier les changements morphologiques des motifs rythmiques de barres d'avant-côte.

\section{Méthodologie}

Les mesures topo-bathymétrique ont été réalisées sur les deux secteurs (Zone Sud et Zone Nord) après chaque épisode de houle important (de l'ordre de $3 \mathrm{~m}$ ) à l'aide d'un GPS cinématique centimétrique (DGPS) et un sondeur mono-faisceau. Les profils sont réalisés tous les $50 \mathrm{~m}$. Ils couvrent l'avant-côte depuis la profondeur $-10 \mathrm{~m}$, jusqu'à la partie haute de la plage (arrière dune), sur les 2 zones évoquées, de $150 \mathrm{~m}$ et $500 \mathrm{~m}$ de linéaire côtier chacune. 13 levés ont ainsi été obtenus durant l'hiver 2008-2009 et le début de l'hiver 2009-2010, 9 d'entres eux couvrent la topographie de la plage.

Sur la totalité des profils levés, un certain nombre d'indicateurs ont été déterminés pour l'étude des évolutions morphologiques : les positions (fronts interne et externe, crête) et formes des barres d'avant-côte ; la position du trait de côte (cote 0 NGF); les volumes des différents compartiments morphologiques (barres, plage émergée, plage immergée). Ces évolutions morphologiques sont confrontées aux forçages météo-marins.

Les données de houle proviennent du houlographe directionnel (bouée datawell, DRELR) positionné au large de Sète $\left(043^{\circ} 22,261^{\prime} \mathrm{N}, 003^{\circ} 46,777^{\prime} \mathrm{E}\right)$ à une profondeur de 30 $\mathrm{m}$. L'évolution du niveau marin provient des relevés du marégraphe (SHOM) dans le port de Sète. Le niveau marin observé, intégrant la surcote, la marée, et la surélévation par les vagues (set-up), sera nommé NM par la suite. Le NM sera exprimé par rapport au 0 hydrographique. Sa valeur moyenne est proche de $+0,40 \mathrm{~m} \mathrm{ZH}$. Les données de vents proviennent d'une station météo proche du site (au niveau de Sète).

Ces données ont permis la caractérisation des épisodes de tempêtes (T), et d'intertempêtes (EPFAs), en terme d'intensité (hauteur des vagues, énergie déployée, niveau marin atteint, vitesse du vent), de direction, mais aussi de durée de l'événement. 


\section{XI $I^{\text {èes }}$ Journées Nationales Génie Côtier - Génie Civil \\ Les Sables d'Olonne, 22-25 juin 2010}

\section{Résultats}

\subsection{Conditions météo-marines et caractéristiques des tempêtes}

Au cours de ce suivi, 5 événements très énergétiques ont eu lieu (cf. figure 3). Ces tempêtes sont restées inférieures à la tempête annuelle définie par le CETMEF (GLOASGUEN, 1998). Ces événements sont séparés par 5 périodes de plus faible agitation, ou "calme" relatif, avec des houles comprises entre 1 et $3 \mathrm{~m}$ (EPFAs). Les caractéristiques hydrodynamiques des différents épisodes sont résumées dans deux tableaux, 1 et 2 . Le premier concerne l'énergie totale cumulée entre les levés (par les EPFAs) et les périodes extra-tempêtes; le second cible l'évènement de plus forte agitation de chaque période inter-levés. Chaque période de calme relatif ou tempête est énumérée, et séparée suivant l'ordre chronologique. L'énumération est la suivante : le "calme" $1(\mathrm{C} 1)$ précède la tempête $1(\mathrm{~T} 1)$, le $\mathrm{C} 2$ précède $\mathrm{T} 2$, et ainsi de suite, ... .

- Caractéristiques des tempêtes majeures

La tempête T1 a eu lieu le 26/12/2008. Elle était caractérisée par une houle d'ESE forte et longue, des brisants "propres" et un faible NM par rapport aux conditions de houle. Cette tempête a eu une période de tombant assez longue, avec un second pic de houle d'ESE courte et ventée. L'énergie totale cumulée lors de cet événement est très importante, avec une forte composante longitudinale.

T2 (+ T2 bis) a eu lieu le 02/02/2009. La houle était plus courte, ventée, de provenance ESE, tournant SE au pic, et le NM enregistré très important $(+0,99 \mathrm{~m})$. T2 est suivie 2 jours après par un EPFA que nous incluons dans l'évènement (T2 bis), bien qu'un relevé morphologique ait été réalisé entre les deux coups. Cette seconde houle de secteur sud est courte, ventée, et la surcote est encore très importante.

T3 a eu lieu le 26/04/2009. Il s'agissait d'une houle courte ventée d'ESE, qui a déployé une énergie avec une forte composante longitudinale. Le NM est resté modéré à faible.

$\mathrm{T} 4$ a eu lieu le 21/10/2009. Il s'agissait d'une houle très forte ( $\mathrm{Hs} \sim 4,5 \mathrm{~m}$ ) mais de courte durée comparée à celle de décembre. La période des vagues est restée moyenne à courte. La provenance sud au montant a tourné à l'ESE au pic d'énergie, et de nouveau au S sur le tombant. Le vent de secteur S-SE était très fort au pic. Le NM enregistré est le maximum de ce suivi $(1,05 \mathrm{~m})$.

T5 a eu lieu le 29/11/2009. Il s'agissait d'une houle courte de sud, avec un vent fort de SSO et un NM modéré à faible. Malheureusement nous n'avons pas pu obtenir les données du houlographe lors du montant de tempête. La durée de la tempête est supposée courte.

- Périodes inter-tempêtes et épisodes de fortes tramontane

Afin de mieux comprendre les évolutions observées, les périodes inter-T ont également été analysées. Certains petits coups de mer (EPFA), non considérés comme des tempêtes, peuvent en effet entraîner des évolutions morphologiques importantes. 
Thème 2 - Dynamique sédimentaire et transports des particules

Tableau 1. Energie totale (Etot, exprimée en mégawatts) de houle au large entre les levés (et composante longitudinale) et caractérisation hydrodynamique des périodes inter-tempête (pré-post T), en considérant le nombre de jours depuis la dernière tempête, le nombre d'évènements de houles modérés (EPFAs), et l'énergie de houle cumulée (Ecmul ; avec sa composante longitudinale, positive=houle de SO). Il est aussi indiqué l'énergie cumulée juste avant la tempête.

\begin{tabular}{|c|c|c|c|c|c|c|c|c|c|c|c|}
\hline $\begin{array}{l}\text { date du } \\
\text { levé }\end{array}$ & $\begin{array}{l}\text { Etot } \\
\text { inter- } \\
\text { levé }\end{array}$ & $\begin{array}{l}\text { Etot } \\
\text { long } \\
\text { inter- } \\
\text { levé }\end{array}$ & $\begin{array}{l}\text { Nom } \\
\text { période } \\
\text { relevée }\end{array}$ & $\begin{array}{l}\text { dernier } \\
\text { evènmt T }\end{array}$ & $\begin{array}{l}\Delta t \\
\text { post-T }\end{array}$ & $\begin{array}{l}\text { EPFA } \\
\text { post-T }\end{array}$ & $\begin{array}{l}\text { EPFA } \\
\text { pré-T }\end{array}$ & $\begin{array}{l}\text { Ecmul } \\
\text { post-T }\end{array}$ & $\begin{array}{l}\text { Ecmul } \\
\text { pré-T }\end{array}$ & $\begin{array}{l}\text { Ecmul } \\
\text { long } \\
\text { post-T }\end{array}$ & $\begin{array}{l}\text { Ecmul } \\
\text { long } \\
\text { pré-T }\end{array}$ \\
\hline UNITES & $M W$ & $M W$ & & date & jours & nbre & nbre & $M W$ & $M W$ & $M W$ & $M W$ \\
\hline $18 / 11 / 08$ & & & & $02 / 11 / 0812 \mathrm{~h}$ & 16.0 & 2 & & 0.691 & & 0.347 & \\
\hline $22 / 12 / 08$ & 3.568 & 1.211 & $C 1$ & $02 / 11 / 0812 \mathrm{~h}$ & 50.0 & 6 & & 4.258 & & 1.558 & \\
\hline 06/01/09 & 8.357 & -1.332 & $T 1$ & $26 / 12 / 0818 h$ & 10.8 & 0 & 6 & 0.219 & 4.265 & 0.008 & 1.559 \\
\hline 13/01/09 & 0.791 & -0.362 & C 2.1 & $26 / 12 / 0818 h$ & 17.8 & 1 & & 1.010 & & -0.353 & \\
\hline 29/01/09 & 0.782 & 0.384 & C 2.2 & $26 / 12 / 0818 h$ & 33.8 & 2 & & 1.791 & & 0.031 & \\
\hline 04/02/09 & 4.166 & -0.487 & $T 2$ & $02 / 02 / 0911 h$ & 2.0 & 0 & 2 & 0.054 & 2.803 & -0.001 & -0.322 \\
\hline 16/02/09 & 2.059 & -1.124 & $T 2$ bis & $02 / 02 / 0911 \mathrm{~h}$ & 14.0 & 1 & & 2.110 & & 1.122 & \\
\hline 12/03/09 & 1.110 & 0.131 & C 3.1 & 02/02/09 11h & 38.0 & 3 & & 3.220 & & 1.253 & \\
\hline $21 / 04 / 09$ & 2.736 & 0.250 & C 3.2 & $02 / 02 / 0911 \mathrm{~h}$ & 78.0 & 6 & & 5.955 & & 1.503 & \\
\hline $30 / 04 / 09$ & 3.067 & -0.970 & $T 3$ & $26 / 04 / 0904 h$ & 4.3 & 0 & 6 & 0.038 & 5.995 & 0.016 & 1.528 \\
\hline 03/11/09 & 7.372 & 0.286 & $C 4+T 4$ & 21/10/09 09h & 13.1 & 0 & 6 & 0.184 & 7.410 & 0.070 & -0.029 \\
\hline $04 / 12 / 09$ & 3.674 & 1.077 & $C 5+T 5$ & $29 / 11 / 0912 \mathrm{~h}$ & 5.0 & 1 & 3 & 0.263 & 2.645 & -0.005 & 0.529 \\
\hline
\end{tabular}

Tableau 2. Caractérisation hydrodynamique des tempêtes ou de l'épisode de houle principal de la période de calme. Les paramètres de houles et NM (par rapport au 0 hydrographique) sont mesurés au pic d'énergie. La durée, l'énergie totale et longitudinale de l'évènement, quand $H s>1 m$, sont indiqués.

\begin{tabular}{|c|c|c|c|c|c|c|c|c|c|c|}
\hline $\begin{array}{l}\text { date du } \\
\text { levé }\end{array}$ & $\begin{array}{l}\text { Nom } \\
\text { période } \\
\text { relevée }\end{array}$ & $\begin{array}{l}\text { évènmt Hs } \\
\text { max période }\end{array}$ & $\begin{array}{l}\text { Hs } \\
\max \end{array}$ & $\begin{array}{l}\text { Ts à } \\
\text { Hs } \\
\max \\
\end{array}$ & $\begin{array}{l}\text { dir. ̀̀ } \\
\text { Hs } \\
\max \end{array}$ & $\begin{array}{l}\text { dir. } \\
\text { norm }\end{array}$ & $\begin{array}{l}\text { NM ̀̀ } \\
\text { Hs } \\
\max \\
\end{array}$ & $\begin{array}{l}\text { durée } \\
\text { évènmt }\end{array}$ & $\begin{array}{l}E \\
\text { évènmt }\end{array}$ & $\begin{array}{l}\text { E long } \\
\text { évènmt }\end{array}$ \\
\hline UNITES & & date & $m$ & $s$ & ${ }^{\circ} N$ & $\circ$ & $m$ & $h$ & $M W$ & $M W$ \\
\hline $18 / 11 / 08$ & & $02 / 11 / 0812 h$ & 3.50 & 7 & 113.9 & -24 & 0.9 & 110 & 5.258 & 1.085 \\
\hline $22 / 12 / 08$ & $C 1$ & $29 / 11 / 0816 h$ & 2.48 & 8 & 180 & 42 & 0.81 & 35 & 1.204 & 0.691 \\
\hline 06/01/09 & $T 1$ & $26 / 12 / 0818 h$ & 3.99 & 10 & 121.1 & -17 & 0.65 & 140 & 8.014 & -1.252 \\
\hline 13/01/09 & $C 2.1$ & 09/01/09 17h & 2.06 & 7 & 116 & -22 & $?$ & 25 & 0.643 & -0.271 \\
\hline $29 / 01 / 09$ & $C 2.2$ & 19/01/09 17h & 1.07 & 7 & 172 & 34 & 0.45 & 21 & 0.253 & 0.150 \\
\hline 04/02/09 & $T 2$ & $02 / 02 / 0911 h$ & 3.30 & 7 & 132.6 & -5 & 0.99 & 87 & 4.062 & -0.499 \\
\hline $16 / 02 / 09$ & T2 bis & 05/02/09 19h & 2.73 & 6 & 181 & 43 & 0.85 & 51 & 1.763 & 1.085 \\
\hline $12 / 03 / 09$ & C 3.1 & $04 / 03 / 0912 h$ & 2.34 & 6 & 184 & 46 & 0.63 & 14 & 0.425 & 0.309 \\
\hline $21 / 04 / 09$ & C 3.2 & 15/04/09 16h & 2.45 & 6 & 147 & 9 & 0.52 & 38 & 0.921 & 0.181 \\
\hline $30 / 04 / 09$ & $T 3$ & 26/04/09 04h & 3.41 & 8 & 119.7 & -18 & 0.68 & 57 & 2.989 & -1.011 \\
\hline 03/11/09 & $C 4+T 4$ & $21 / 10 / 0909 h$ & 4.50 & 8 & 121 & -17 & 1.05 & 69 & 4.500 & 0.262 \\
\hline $04 / 12 / 09$ & $C 5+T 5$ & $29 / 11 / 0912 \mathrm{~h}$ & 3.20 & 8 & 179 & 41 & 0.62 & 16 & 0.950 & 0.624 \\
\hline
\end{tabular}

Sur les 2 tableaux ci-dessus, les tempêtes sont surlignées en gris. Les valeurs écrites en vert indiquent un fonctionnement partiel du houlographe, et donc des données incomplètes (période estivale et montant de la tempête du 29 novembre 2009). 
Le calme 1 (C1), du 18/11/2008 au 22/12/2008 (date des levés), a été caractérisé par des houles de faible amplitude, des conditions peu ventées et à NM bas dans l'ensemble. Toutefois, un total de 6 houles successives de SE, moyennes à faibles, a été observé. 2 évènements se détachent car ils correspondent à des périodes de vagues anormalement importantes, respectivement de $10 \mathrm{~s}(\mathrm{E})$ et $8 \mathrm{~s}(\mathrm{~S})$.

C2.1, du 06/01/2009 au 13/01/2009, présente un seul épisode de houle d'E moyennement haute, longue et propre.

C2.2, du 13/01/2009 au 29/01/2009, ne montre qu'un EPFA, où Hs $\approx 1 \mathrm{~m}$. Il est cependant caractérisé par un évènement de tramontane intense le 24 janvier.

C3.1, du 16/02/2009 au 12/03/2009. La période est caractérisée par 2 houles courtes et ventées de provenance $\mathrm{S}$ à $\mathrm{SE}$ pour des NM moyens, mais aussi par un épisode de tramontane très fort le 04 mars.

C3.2, du 12/03/2009 au 21/04/2009, est une longue période très calme. Cependant 3 épisodes de houles moyennes, courtes, et ventées ont eu lieu, avec des NM modérés.

C4, précède T4 (et le levé du 03/11) et comprend toute la période estivale où la bouée n'a pas fonctionnée. Un levé lidar (topographie et bathymétrie laser aéroportée) a été effectué en août, mais il n'a pas pu être utilisé ici. Les modèles de prévisions de houle supposeraient l'existence de 6 EPFA, faibles à modérés, correspondant à des conditions majoritairement ventées avec un NM moyen à assez haut.

C5, précède T5. Il n'y a, là aussi, pas de levé pré-tempête qui individualise C5 de T5. C5 décrit 2 houles courtes de SSE pour des NM moyens, comme lors de T5.

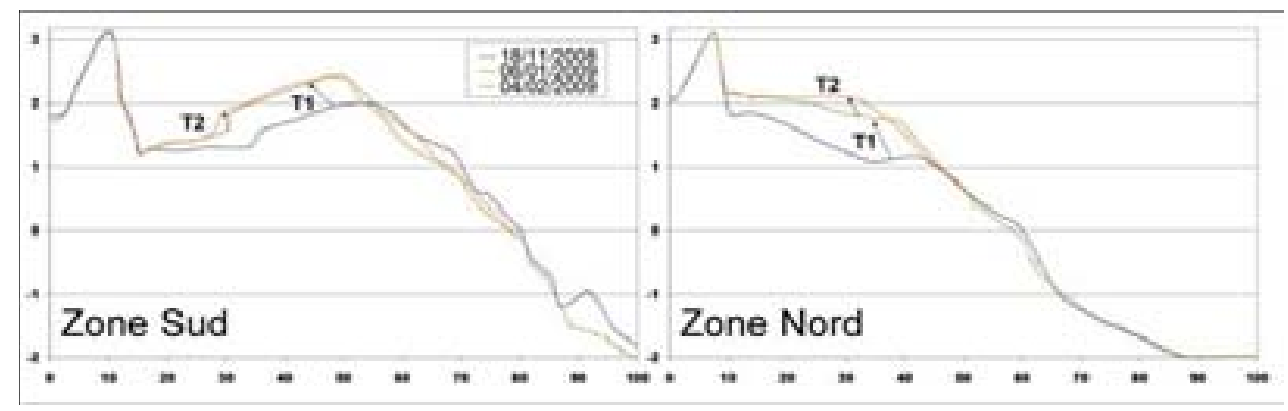

Figure 2. Dépôt massif de sable en haut de plage lors des événements de tempêtes de décembre 2008(T1) et février 2009 (T2) (coupe transversale de la plage, unités métriques, altitudes selon le référenciel IGN69).

\subsection{Réponse morphologique aux événements de tempête}

De manière générale, les tempêtes les plus importantes se caractérisent par un dépôt massif de sable en haut de plage (figure 2). Dès que Hs dépasse $\sim 3 \mathrm{~m}$, ce qui est variable selon la surcote, le jet de rive surpasse la berme pourtant très haute sur la zone sud, et atteint le front dunaire. Ce phénomène a été observé sur les deux secteurs (figure 2). La figure 4 (en bas) fait clairement apparaître un seuil, dans la hauteur significative de la houle, à partir duquel la plage est engraissée. Ce seuil semble être situé à environ 
Thème 2 - Dynamique sédimentaire et transports des particules

2,9-3 m, même si l'effet du NM reste à estimer. Ces apports peuvent-êtres très importants (des dépôts atteignant près de un mètre ont été observés en pied de dune) et surpassent apparemment l'érosion du bas de plage qui peut parfois se produire.

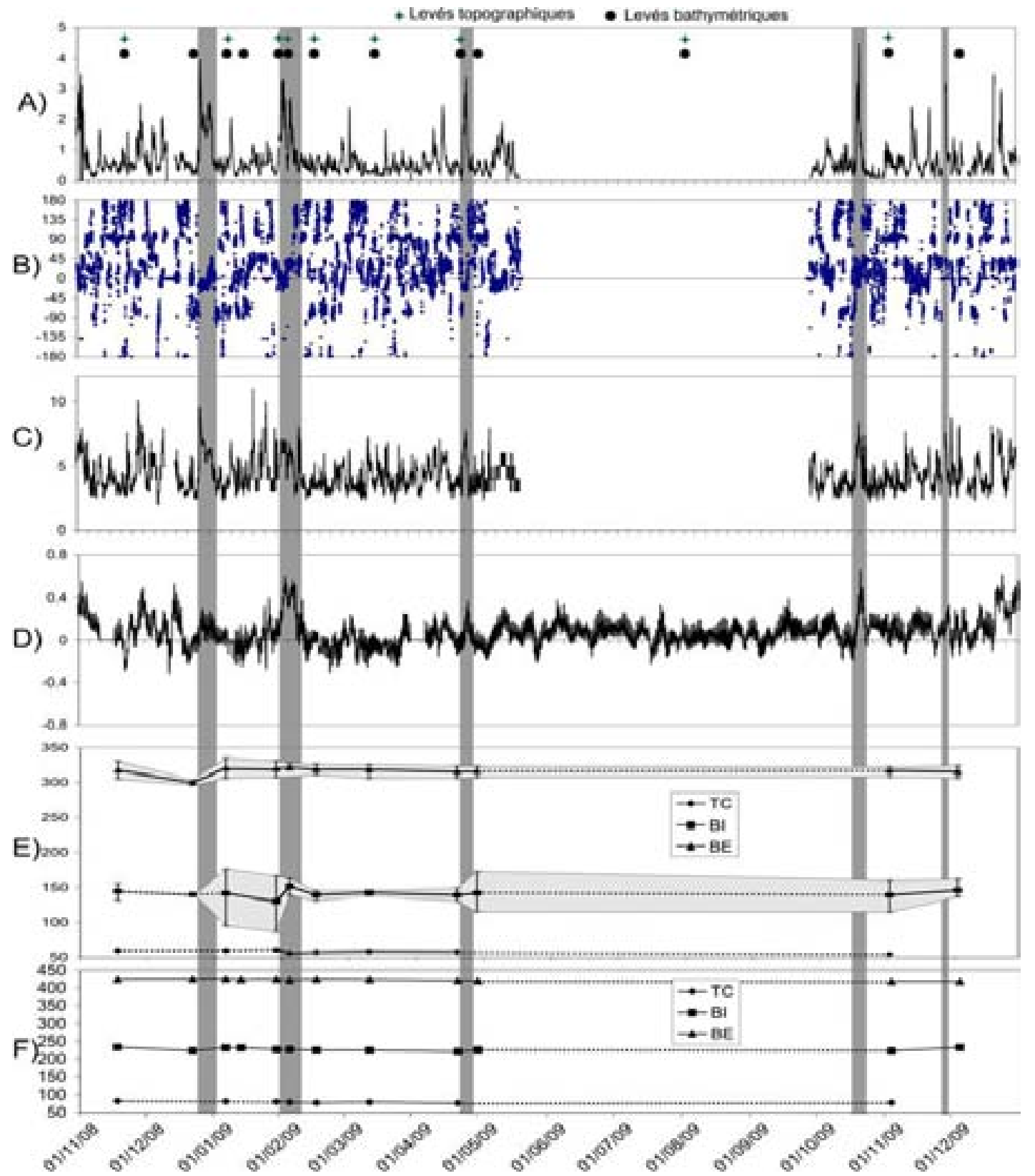

Figure 3. Hydrodynamique et évolutions morphologiques lors de la période de mesures. A) Hauteur significative de la houle (en $\mathrm{m}$ ); B) Angle d'incidence de la houle au large (positif côté $N E$ ) (en degrés) ; C) Période significative (en s) ; D) niveau marin (en $m$ par rapport au zéro hydrographique); E) Zone Nord: position moyenne et déviation standard du trait de côte (TC), de la barre interne (BI) et de la barre externe (BE) ;

$F) Z o n e ~ S u d$ : position moyenne et déviation standard du TC, BI et BE. 


\section{XI $I^{\text {èmes }}$ Journées Nationales Génie Côtier - Génie Civil}

Les Sables d'Olonne, 22-25 juin 2010

Les épisodes de fortes tramontanes provoquent à l'inverse, en l'absence totale de houle, des décapages de la plage vers le talus de plage immergé, à des vitesses verticales d'érosions qui atteindraient d'après nos mesures $1 \mathrm{~cm}$ par heure sur le haut de plage.

Lors des tempêtes, on observe également un recul de la barre interne (BI) (cf. figure 3-E et F). A l'instar du volume de la plage il semblerait aussi que les déplacements transversaux de la barre d'avant-côte interne dans son ensemble soient conditionnés par la hauteur de houle atteinte lors des évènements entre les levés (Hs max). La barre recule lorsque la houle dépasse un seuil de l'ordre de 2,7-3,0 $\mathrm{m}$ de Hs, et avance lorsque les houles sont comprises entre 1,0 et 2,7 m (EPFA), et ce sur les deux zones (figure 4). Seule T2 n'a que peu d'effet sur la barre interne au sud. Lors des tempêtes la barre externe semble aussi s'affaisser.

Ces comportements (recul de la barre et dépôt en haut de plage) sont observés sur les deux secteurs, malgré des morphologies d'avant-côte très différentes. Les vitesses de migration et les ampleurs de variations de volume semblent directement corrélées avec la hauteur significative atteinte pendant la tempête.

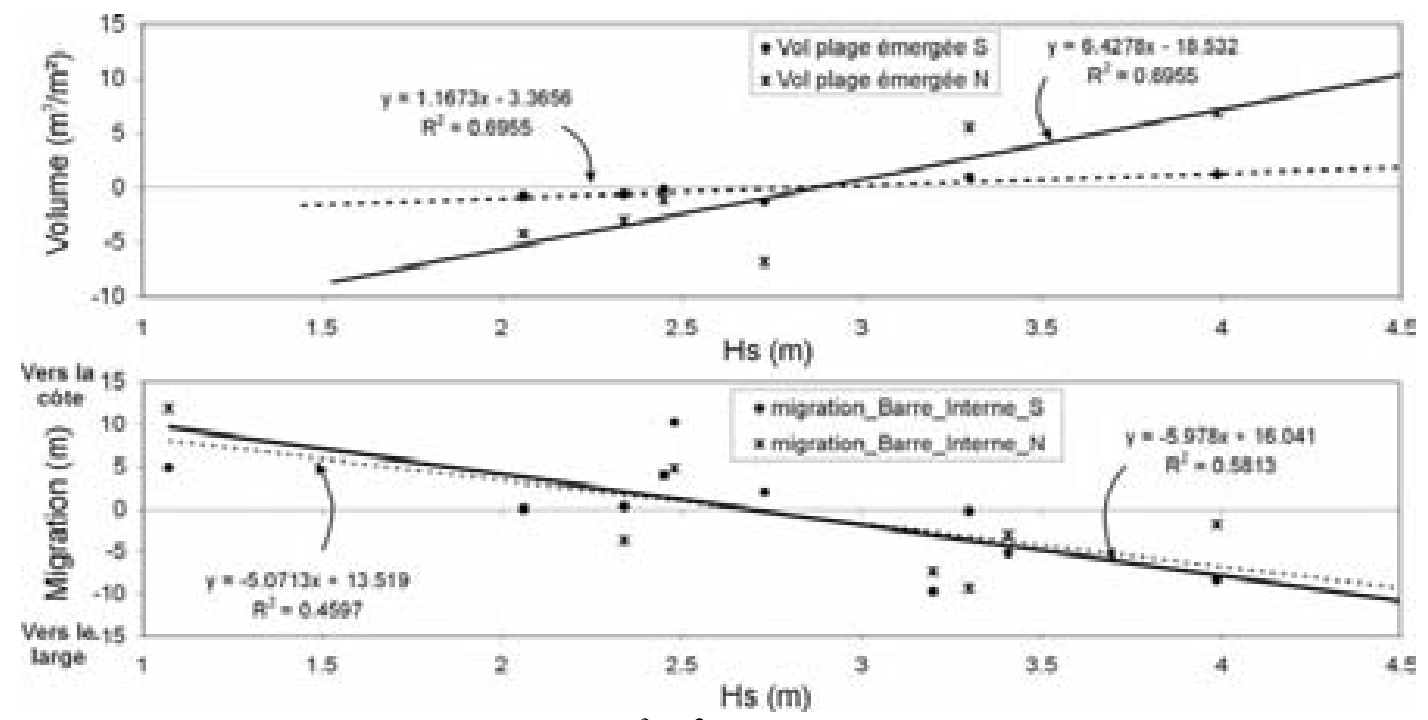

Figure 4. Variation de volume $\left(0.01 \mathrm{~m}^{3} / \mathrm{m}^{2}\right)$ de la plage émergée (en haut) et migration de la distance moyenne de la crête de la barre interne sur chaque zone (en bas) en fonction de la hauteur significative de la houle au pic de la tempête.

\subsection{Dynamique tridimensionnelle des barres lors des tempêtes}

Sur la figure 3-E et F, l'enveloppe grisée présente l'écart maximal entre la position des crêtes sur les différents profils de la zone, et la position moyenne de la crête (invisible sur 3-F). Ceci traduit donc une variabilité latérale de la position de la crête et constitue donc un bon indicateur de la tridimensionnalité ou de l'obliquité de la barre. On observe donc, dans la zone Nord, une forte variabilité de la tridimensionnalité de la barre, et un changement rapide d'un état morphologique à un autre lors des tempêtes (par exemple 
d'une barre longitudinale à une barre en croissant ou inversement.). Ainsi, la BI devient d'avantage tri-dimensionnelle lors de T1 et T3, alors qu'elle se linéarise lors de T2. La figure 5 présente les bathymétries pré- et post-tempête dans la zone Nord pour les 5 événements majeurs observés lors de la campagne de mesures.

Lors de T1, la BI en festons très bien formés s'est rompue au niveau des points bas (ventres), qui reculent, tandis que les points hauts (cornes) ont migré vers la côte. L'un des deux se trouve maintenant soudé à la plage ce qui explique la variabilité longitudinale de la barre (figure 4). La morphologie de la fosse, oblique, suggère un chenal transverse d'évacuation de l'eau dans le sens préférentiel de la dérive. Lors de $\mathrm{T} 2$, d'incidence plus frontale, les points hauts s'émoussent et une barre ainsi qu'une fosse linéaire se reforment. La barre est globalement linéaire, même si des points plus hauts se distinguent. Lors de T3, cette BI linéaire à 2 points hauts est modifiée en un système dissymétrique de barre en festons de grandes ampleurs, fortement asymétriques. La fosse est creusée préférentiellement du côté SO de la corne, tandis qu'à son extrémité la ligne de crête de la barre est basse (ventre). Cette réponse morphologique serait semblable aux observations de CERTAIN (2002) d'après les campagnes 1994 et 1999 sur le même site. Lors de T4, après la période estivale, la réaction est similaire à celle de $\mathrm{T} 1$. La $\mathrm{BI}$ en feston est percée au niveau de son ventre, tandis que la corne du feston avance et s'attache à la côte sous forme d'un point haut à morphologie complexe, où l'on devine l'enracinement de la fosse au niveau de l'attache de ce point. Pour finir, la tempête 5 en provenance du Sud, modifie totalement la morphologie car une barre linéaire est de nouveau créée.

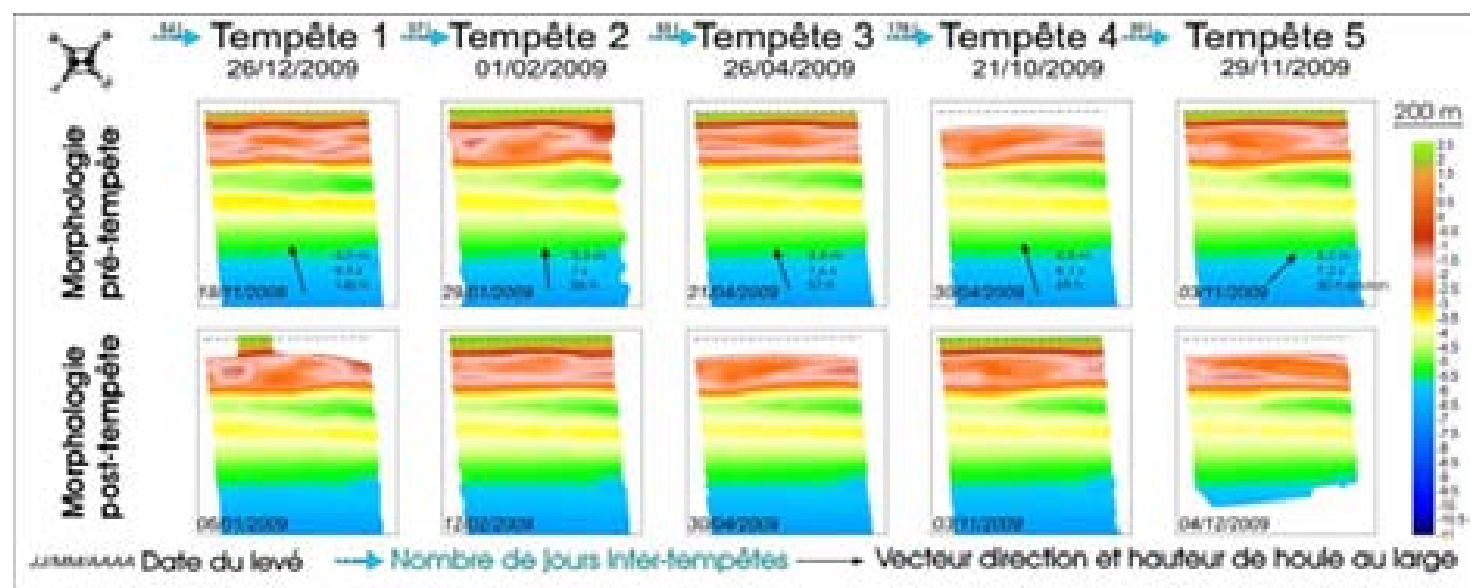

Figure 5. Bathymétries pré- et post-tempêtes dans la zone nord.

\section{Discussion : impacts des facteurs de forçages sur la réponse morphologique}

Les résultats obtenus sur plus d'un an d'observation des réponses morphologiques aux coups de mer mettent en évidence la relation entre l'intensité des forçages météo-marins et l'évolution des plages. De manière générale, la BI recule dès lors que Hs augmente, à 


\section{XI $I^{\text {èes }}$ Journées Nationales Génie Côtier - Génie Civil \\ Les Sables d'Olonne, 22-25 juin 2010}

l'exception de la T2. Lors de cette tempête, le NM était très haut $(0,99 \mathrm{~m})$, la surcote très importante, et la modification du comportement peut sans doute s'expliquer par la diminution de la hauteur relative $\mathrm{Hs} / \mathrm{d}$, où $\mathrm{d}$ est la hauteur d'eau au dessus de la barre. Par ailleurs, la durée entre les deux épisodes (T1 et T2) est très courte $(37 \mathrm{j})$, avec très peu de houles constructives (EPFAs), et il est probable que T2 soit survenue sur un système toujours en "déséquilibre".

Dans la zone sud les évolutions sont moins contrastées que dans la zone nord. Dans ce secteur, rappelons "témoin", car les barres y sont linéaires, on observe une migration vers le large de BI lors des événements les plus importants, mais pour des évènements d'énergie plus modérée, la barre migre vers la plage très rapidement. En comparant l'impact des périodes de calme C1 et C3 avec C2, l'effet des épisodes de "petites" houles à longues périodes, par rapports à ceux à courtes périodes, entraînerait un retour plus rapide aux conditions pré-tempêtes, ce qui n'est pas observé avec autant de clarté dans le secteur nord.

Les résultats obtenus montrent donc qu'il semble exister un seuil à partir duquel les comportements changent. Ce seuil est principalement associé à la hauteur significative de la houle lors du pic de tempête. Ainsi, on obtient une relation directe entre la hauteur des vagues et la migration des barres vers le large, et la hauteur des vagues et les gains volumétriques sur la plage. Les tempêtes entraîneraient aussi une perte de volume des barres, bien que cela soit plus difficile à montrer sur la zone au nord où les morphologies 3D peuvent migrer longitudinalement avec la dérive. Il existe donc une relation bien établie entre l'intensité des forçages et les réponses morphologiques en termes de mobilité sédimentaire (variations de volumes, et intensité des déplacements sableux), et de manière inattendue les apports de sable sur le haut de plage sont à relier aux évènements de tempête.

En revanche, les motifs de barres, en particulier dans la zone nord, ne semblent pas répondre directement aux forçages météo-marins, mais d'avantage à la morphologie préexistante avant la tempête. En effet, T2 et T3, qui sont relativement similaires en termes de hauteur de vagues et de direction, ont eu des réponses morphologiques très distinctes : de festons détruits vers une barre linéaire pour T2 ; d'une barre linéaire vers des festons très marqués pour T3.

Les épisodes de tramontane pourraient jouer un rôle prépondérant en l'absence de vagues à Sète, car ils provoqueraient un retour du sable du haut de plage vers la zone de jet de rive, comme il a était montré par DURAND (1999) plus au sud du Golfe du Lion.

\section{Conclusions}

Les campagnes de terrain réalisées depuis novembre 2008 ont permis d'obtenir une base de données inédite et des informations quantitatives sur les réponses morphologiques lors d'événements énergétiques successifs. Ces levés ont été réalisés à chaque coup de 
mer, permettant d'analyser la contribution spécifique de chacun des événements, mais également celle des phases de reconstruction.

Les résultats semblent démontrer que, lors de chaque événement, les différents forçages (particulièrement la hauteur significative atteinte par la houle) jouent un rôle direct sur la dynamique sédimentaire (mobilité des fonds, volumes, ...), mais ne semblent pas jouer un rôle prédominant sur les motifs des barres. La dynamique des barres parait fortement influencée par la morphologie préexistante avant la tempête.

Même si l'échantillonnage reste limité, les résultats obtenus semblent démontrer l'importance de la morphologie pré-tempête sur la dynamique des barres d'avant-côte lors des épisodes extrêmes, et démontre aussi le rôle essentiel des périodes de plus faibles houles.

\section{Remerciements}

Ce travail a été réalisé dans le cadre du projet FP7 MICORE n²02798. Le premier auteur bénéficie d'une allocation doctorale de la Région Languedoc-Roussillon et du BRGM. Merci à Gwenaëlle Bodéré, Cécile Godon et Pierre Ferrer pour leur contribution aux mesures de terrain.

\section{Références bibliographiques}

AKOUANGO E. (1997). Morphodynamique et dynamique sédimentaire dans le Golfe du Lion. Contribution à l'étude de la zone côtière dans l'actuelle et le quaternaire récent. Thèse de Doctorat, Université de Perpignan, $200 \mathrm{p}$.

BARUSSEAU J.P, SAINT-GUILY B. (1981). Disposition, caractères et mode de formation des barres d'avant-côte festonnées du littoral du Languedoc-Roussillon (France). Oceanologica Acta., 4, 3, pp 297-304.

CERTAIN R. (2002). Morphodynamique d'une côte sableuse microtidale à barres : le Golfe du Lion (Languedoc-Roussillon). Thèse de Doctorat, Université de Perpignan, $109 \mathrm{p}$.

CERTAIN R., BARUSSEAU J.P (2005). Conceptual modelling of sand bars morphodynamics for a microtidal beach (Sète, France). Bulletin de la Société géologique de France, 2005, t. 176, no 4, pp 343-354.

DURAND P. (1999). L'évolution des plages de l'ouest du Golfe du Lion au XXème siècle. Thèse de doctorat en géographie physique, Université Lumière Lyon 2, 462 p.

FERRER P., REY V., CERTAIN R., ADLOFF F., MEULÉ S. (2006). Les ondes infragravitaires et leur rôle éventuel dans la formation de croissants de plage : cas de la plage de Sète. IXèmes Journées Nationales Génie Côtier-Génie Civil, Brest, pp 187194. doi:10.5150/jngcgc.2006.019-F

GLOASGUEN G. (1998). Estimation de la période de retour de la tempête du 16 décembre 1997 à Sète. Rapport STNMTE, CETMEF, 10 p. 